\title{
Design Single Fuzzy Adaptive Controller for 4 DOF Medical Robots
}

\author{
Hossein Rashidi Bod ${ }^{1}$, Farzin Piltan ${ }^{1}$, Somayeh Jowkar ${ }^{1}$, Amirzubir Sahamijoo ${ }^{1}$, \\ Ali Taghizadegan ${ }^{1}$, Rouhollah Bahrami ${ }^{1}$ and Nasri. B Sulaiman ${ }^{1,2}$ \\ ${ }^{1}$ Intelligent System and Robotic Lab, Iranian Institute of Advanced Science and \\ Technology (IRAN SSP), Shiraz/Iran \\ ${ }^{2}$ Department of Electrical Engineering, Faculty of Engineering, University Putra \\ Malaysia, Malaysia \\ piltan_f@iranssp.org,www.iranssp.org/english
}

\begin{abstract}
This research paper focuses on the design and analysis of a high performance adaptive $P D$ like fuzzy computed Torque controller for second order nonlinear uncertain (medical robot manipulator) system, in presence of uncertainties. The proposed approach effectively combines of design methods from Computed Torque controller (CTC), adaptive controller and fuzzy logic theory to improve the performance, stability and robustness of the CTC controller.

Computed Torque Controller has two important challenges, system's dynamic dependency and stability challenge. The nonlinearity term of the CTC is used to eliminate the decoupling and nonlinear term of link's dynamic parameters. However, nonlinearity term of CTC is very essential to reliability but in uncertain condition or highly nonlinear dynamic systems, it can cause some problems. To solve this challenge the PD fuzzy logic controller is used as a model-based PD like fuzzy computed torque controller. The PD like fuzzy computed torque controller is updated based on online tuning coefficients. In order to reduce the online computation burden, the PD like fuzzy logic controller is also used to coefficients online tuning. As a result, in proposed method fuzzy logic controller is used to dynamic estimation and online tuning and as a result, improves the stability. This controller improves the stability and robustness, reduces the error as well and reduces the level of energy due to the torque performance as well.
\end{abstract}

Keywords: surgical robots, four degrees of freedom, computed torque controller, fuzzy logic theory, PD like fuzzy logic, adaptive technique

\section{Introduction}

Robot manipulators have many applications in aerospace, manufacturing, automotive, medicine and other industries. Robot manipulators consist of three main parts:

- Mechanical

- Electrical

- control.

In the mechanical point of view, robot manipulators are collection of serial or parallel links, which have connected by revolute and/or prismatic joints between base and endeffector frame. The robot manipulators electrical parts are used to run the controllers, actuators for links motion and sensors, which including the following subparts: power supply to supply the electrical and control parts, power amplifier to amplify the signal and driving the actuators, DC/stepper/servo motors or hydraulic/pneumatic cylinders to move the links, and transmission part to transfer data between robot manipulator subparts [1]. 
Control part is used to adjust the timing between the subparts of robot manipulator to reach the best trajectory. It provides four main abilities in robot manipulators [1, 4]:

- controlling the manipulators movement in correct workspace

- sensing the information from the environment

- being able to intelligent control behavior

- Processing the data and information between all subparts

In this research paper we introduced, basic concepts of medical robot manipulator and control methodology. Our research medical robot manipulator is a 4 DOF serial robot manipulator. From the control point of view, robot manipulator divides into two main parts i.e. kinematics and dynamic parts. The dynamic parameters of this system are highly nonlinear. To control of this system nonlinear control methodology (computed torque controller) is introduced. A controller (control system) is a device, which cans sense information from system to improvement the dynamic behavior of system based on actuation and computation. From scientific perspectives, control theory is divided into two parts:

- linear control theory

- nonlinear control theory

In this research nonlinear control theory is used to control of 4-DOF medical robot. Nonlinear control theory is also has two main divisions;

- Conventional control theory

- Soft computing control theory

Conventional nonlinear control theories are highly sensitive to system's behavior and work based on cancelling decoupling and nonlinear terms of dynamic parameters of each likes in robot manipulators. These controllers divided into five groups [1-3]:

- Feedback linearization (computed-torque control)

- passivity-based control

- $\quad$ sliding mode control (variable structure control)

- Backstepping control

- Lyapunov-based control

Computed Torque Controller (CTC) is one of the effective nonlinear controllers to control of robot manipulator [4-5]. Consequently, to have a good performance, linearization and decoupling without using many gears, feedback linearization (computedtorque) control methodologies is presented. To design computed torque controller, an accurate dynamic model of robot manipulator plays an important role. To modelling an accurate dynamic system, modelling of complex parameters is needed to form the structure of system's dynamic model. It may be very difficult to include all the complexities in the system dynamic model [6].

Soft computing control theory is used to modelling and control. This type of theory is divided into the following methodologies:

- Fuzzy logic theory

- Neural network theory

- Genetic algorithm theory

- Neuro-fuzzy theory

In recent years, artificial intelligence theory has been used to design system's controller. Neural network, fuzzy logic and neuro-fuzzy are synergically combined with nonlinear classical controller and used in nonlinear, time variant and uncertain plant (e.g., robot manipulator). Fuzzy logic controller (FLC) is one of the most important applications of fuzzy logic theory. This controller can be used to control nonlinear, uncertain, and noisy systems. This method is free of some model techniques as in model-based controllers. As mentioned that fuzzy logic application is not only limited to the modelling of nonlinear systems but also this method can help engineers to design a model-free controller. In this research, single adaptive PID like fuzzy controller is design, estimate, improve the 
response of PD computed torque controller, and applied to 4-DOF surgical robot to improve the sensitivity [7-8].

This paper is organized as follows; second part (theory), focuses on the system modeling dynamic formulation, Design computed Torque Controller and fundamental of fuzzy logic controller. Third part is focused on the methodology to design single PID like adaptive computed torque controller. The forth part is focused on results and discussion. The last part focuses on the conclusion.

\section{Theory}

System's Dynamic: A dynamic function is the study of motion with regard to the forces. Dynamic modeling of surgical robot manipulators is used to illustrate the behavior of robot manipulator (e.g., nonlinear dynamic behavior), design of nonlinear conventional controller and for simulation. It is used to analyses the relationship between dynamic functions output (e.g., joint motion, velocity, and accelerations) to input source of dynamic functions (e.g., force/torque or current/voltage). Dynamic functions is also used to explain the some dynamic parameter's effect (e.g., inertial matrix, Coriolios, Centrifugal, and some other parameters) to system's behavior [1].

The equation of multi degrees of freedom (DOF) surgical robot manipulator dynamics is considered by the following equation[1]:

$$
[A(\boldsymbol{q})] \ddot{\boldsymbol{q}}+[N(\boldsymbol{q}, \dot{\boldsymbol{q}})]=[\boldsymbol{\tau}]
$$

The dynamic formulations for 4 Degrees of Freedom serial links surgical robot manipulator are computed by;

$$
A(\ddot{\theta})\left[\begin{array}{c}
\ddot{\theta}_{1} \\
\ddot{\theta}_{2} \\
\ddot{\theta}_{3} \\
\ddot{\theta}_{4}
\end{array}\right]+B(\theta)\left[\begin{array}{c}
\dot{\theta}_{1} \dot{\theta}_{2} \\
\dot{\theta}_{1} \dot{\theta}_{3} \\
\dot{\theta}_{1} \dot{\theta}_{4} \\
\dot{\theta}_{2} \dot{\theta}_{3} \\
\dot{\theta}_{2} \dot{\theta}_{4} \\
\dot{\theta}_{3} \dot{\theta}_{4}
\end{array}\right]+C(\theta)\left[\begin{array}{c}
\dot{\theta}_{1}^{2} \\
\dot{\theta}_{2}^{2} \\
\dot{\theta}_{3}^{2} \\
\dot{\theta}_{4}^{2}
\end{array}\right]+G(\theta)=\left[\begin{array}{c}
\tau_{1} \\
\tau_{2} \\
\tau_{3} \\
\tau_{4}
\end{array}\right]
$$

Computed Torque Controller: Computed torque controller (CTC) is one of the effective nonlinear control methodologies for second order nonlinear system (e.g., robot manipulator). Computed torque controller works based on behavior (dynamic formulation) of robot manipulator which caused to works very well when all dynamic and physical parameters are known. In uncertain dynamic parameters when the robot manipulator has variation computed torque controller has challenges. In this type of controller, if the desired position trajectory for the manipulator defined as $\boldsymbol{q}_{\boldsymbol{d}}(\boldsymbol{t})$, and the actual position trajectory defined as $\boldsymbol{q}_{\boldsymbol{a}}(\boldsymbol{t})$, the tracking error calculated by [1-4];

$$
e(t)=q_{d}(t)-q_{a}(t)
$$

Based on this research the errors for 4 links are;

If state space equation is defined by;

$$
\left[\begin{array}{l}
e_{1} \\
e_{2} \\
e_{3} \\
e_{4}
\end{array}\right]=\left[\begin{array}{l}
q_{d_{1}}(t)-q_{a_{1}}(t) \\
q_{d_{2}}(t)-q_{a_{2}}(t) \\
q_{d_{3}}(t)-q_{a_{3}}(t) \\
q_{d_{4}}(t)-q_{a_{4}}(t)
\end{array}\right]
$$

$$
\dot{x}=A x+B U
$$


According to the Brunousky canonical form $\boldsymbol{U}$ is the nonlinearity term and defined by;

$$
U=\ddot{\boldsymbol{q}}_{d}+\boldsymbol{A}^{-1}(\boldsymbol{q}) \cdot\{\boldsymbol{N}(\boldsymbol{q} \cdot \dot{\boldsymbol{q}})-\boldsymbol{\tau}\}
$$

Based on (5) and (6)

$$
\dot{x}=\left[\begin{array}{ll}
\mathbf{0} & I \\
\mathbf{0} & \mathbf{0}
\end{array}\right] \boldsymbol{x}+\left[\begin{array}{l}
\mathbf{0} \\
I
\end{array}\right] \boldsymbol{U}
$$

According to $x=\left[e^{T} \dot{e}^{T}\right]^{T}$

$$
\frac{d}{d t}\left[\begin{array}{l}
e \\
\dot{e}
\end{array}\right]=\left[\begin{array}{ll}
0 & I \\
0 & 0
\end{array}\right] \cdot\left[\begin{array}{l}
e \\
\dot{e}
\end{array}\right]+\left[\begin{array}{l}
0 \\
I
\end{array}\right] U
$$

Based on (60), the requirement torque calculated by;

$$
\tau=A(q)\left(\ddot{q}_{d}+U\right)+N(\dot{q}, q)
$$

According to nonlinear feed-back control low, (9) is nonlinear food-back controller and it is used to tracking of desired trajectory. According to the literature [7], $\mathrm{U}$ is the linear part of this nonlinear controller and the PD, PI or PID terms can be selected. The formulation of $\mathrm{PD}$ computed torque controller is;

$$
\tau=A(q)\left(\ddot{q}_{d}+K_{v} \dot{e}+K_{p} e\right)+N(q, \dot{q})
$$

Where $\boldsymbol{K}_{\boldsymbol{p}}, \boldsymbol{K}_{\boldsymbol{v}}$ are the controller gain coefficients. This type of controller has two main important parts; partly linear part and nonlinear term of dynamic equivalent part.

Fuzzy Logic Controller: Supposed that $U$ is the universe of discourse and $x$ is the element of $U$, therefore, a crisp set can be defined as a set which consists of different elements $(x)$ will all or no membership in a set. A fuzzy set is a set that each element has a membership grade, therefore it can be written by the following definition [7];

$$
\boldsymbol{A}=\left\{\boldsymbol{x}, \mu_{A}(\boldsymbol{x}) \mid \boldsymbol{x} \in X\right\} ; \boldsymbol{A} \in \boldsymbol{U}
$$

Where an element of universe of discourse is $x, \mu_{A}$ is the membership function (MF) of fuzzy set. The membership function $\left(\mu_{A}(x)\right)$ of fuzzy set $A$ must have a value between zero and one. If the membership function $\mu_{A}(x)$ value equal to zero or one, this set change to a crisp set but if it has a value between zero and one, it is a fuzzy set. Defining membership function for fuzzy sets has divided into two main groups; namely; numerical and functional method, which in numerical method each number has different degrees of membership function and functional method used standard functions in fuzzy sets. The membership function which is often used in practical applications includes triangular form, trapezoidal form, bell-shaped form, and Gaussian form. A Trapezoidal membership function of fuzzy set is defined by the following equation

$$
\boldsymbol{\mu}_{\boldsymbol{F}(\boldsymbol{x})}=\left\{\begin{array}{cl}
\frac{\boldsymbol{x}-\boldsymbol{a}}{\boldsymbol{x}-\boldsymbol{a}}, & x<a \\
\frac{\boldsymbol{d}-\boldsymbol{x}}{\boldsymbol{d}-\boldsymbol{c}}, & c \leq x<d \\
\mathbf{0}, & x>d
\end{array}\right.
$$

A Triangular membership function of fuzzy set is defined by the following equation

$$
\boldsymbol{\mu}_{\boldsymbol{F}(\boldsymbol{x})}=\left\{\begin{array}{cl}
\frac{\boldsymbol{x}-\boldsymbol{a}}{\boldsymbol{\boldsymbol { b }}-\boldsymbol{a}}, & x<x<b \\
\frac{\boldsymbol{c}-\boldsymbol{x}}{\boldsymbol{c}-\boldsymbol{b}}, & b \leq x \leq c \\
\mathbf{0}, & x>c
\end{array}\right.
$$

A Gaussian membership function of fuzzy set is defined by 


$$
\mu_{F(x)}=e^{\frac{-\left(x-c_{F}\right)^{2}}{W}}
$$

and a Bell-shaped membership function of fuzzy set is defined by

$$
\mu_{F(x)}=\frac{1}{1+\left(x-c_{F}\right)^{2}}
$$

Linguistic variable can open a wide area to use of fuzzy logic theory in many applications (e.g., control and system identification). In a natural artificial language all numbers replaced by words or sentences. In Figure 1 the linguistic variable is torque and the linguistic values are Low,Medium and High.

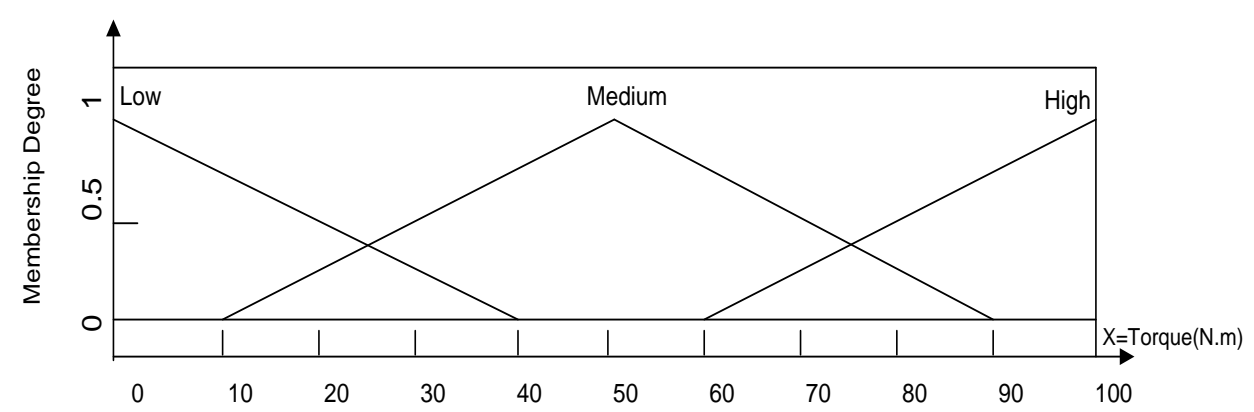

Figure 1. Linguistic Variable and Linguistic Value

If - then Rule statements are used to formulate the condition statements in fuzzy logic. A single fuzzy If - then rule can be written by

$$
\text { If } x \text { is } A \text { Then } y \text { is } B
$$

where $A$ and $B$ are the Linguistic values that can be defined by fuzzy set, the $I f$ - part of the part of " $x$ is $A$ " is called the antecedent part and the then - part of the part of " $y$ is $B$ " is called the Consequent or Conclusion part. The antecedent of a fuzzy if-then rule can have multiple parts, which the following rules shows the multiple antecedent rules:

$$
\text { if } e \text { is NB and } \dot{e} \text { is ML then } T \text { is } L L
$$

where $e$ is error, $\dot{e}$ is change of error, $N B$ is Negative Big, $M L$ is Medium Left, $T$ is torque and $L L$ is Large Left.

If - then rules have three parts, namely, fuzzify inputs, apply fuzzy operator and apply implication method which in fuzzify inputs the fuzzy statements in the antecedent replaced by the degree of membership, apply fuzzy operator used when the antecedent has multiple parts and replaced by single number between 0 to 1 , this part is a degree of support for the fuzzy rule, and apply implication method used in consequent of fuzzy rule to replaced by the degree of membership.

The fuzzy inference engine offers a mechanism for transferring the rule base in fuzzy set which it is divided into two most important methods, namely, Mamdani method and Sugeno method. Mamdani method is one of the common fuzzy inference systems and he designed one of the first fuzzy controllers to control of system engine. Mamdani's fuzzy inference system is divided into four major steps: fuzzification, rule evaluation, aggregation of the rule outputs and defuzzification. Michio Sugeno use a singleton as a membership function of the rule consequent part. The following definition shows the Mamdani and Sugeno fuzzy rule base 


\section{Mamdani \\ Sugeno \\ $F . R^{1}:$ if $\quad x$ is $A$ and $y$ is $B$ then $\quad z$ is $C$ \\ $F . R^{1}$ :if $x$ is $A$ and $y$ is $B$ then $f(x, y)$ is $C$}

When $x$ and $y$ have crisp values fuzzification calculates the membership degrees for antecedent part. Rule evaluation focuses on fuzzy operation $(A N D / O R)$ in the antecedent of the fuzzy rules. The aggregation is used to calculate the output fuzzy set and several methodologies can be used in fuzzy logic controller aggregation, namely, Max-Min aggregation, Sum-Min aggregation, Max-bounded product, Max-drastic product, Maxbounded sum, Max-algebraic sum and Min-max. Two most common methods that used in fuzzy logic controllers are Max-min aggregation and Sum-min aggregation. Max-min aggregation defined as below

$$
\mu_{U}\left(x_{k}, y_{k}, U\right)=\mu_{\cup_{i=1}^{r} F R^{i}}\left(x_{k}, y_{k}, U\right)=\max \left\{\min _{i=1}^{r}\left[\mu_{R} p q\left(x_{k}, y_{k}\right), \mu_{p_{m}}(U)\right]\right\}
$$

The Sum-min aggregation defined as below

$$
\mu_{U}\left(x_{k}, y_{k}, U\right)=\mu_{\cup_{i=1}^{r} F R^{i}}\left(x_{k}, y_{k}, U\right)=\sum \min _{i=1}^{r}\left[\mu_{R} p q\left(x_{k}, y_{k}\right), \mu_{p_{m}}(U)\right]
$$

where $r$ is the number of fuzzy rules activated by $x_{k}$ and $y_{k}$ and also $\mu_{\cup_{i=1}^{r} F R^{i}}\left(x_{k}, y_{k}, U\right)$ is a fuzzy interpretation of $i-t h$ rule. Defuzzification is the last step in the fuzzy inference system which it is used to transform fuzzy set to crisp set. Consequently defuzzification's input is the aggregate output and the defuzzification's output is a crisp number. Centre of gravity method (COG) and Centre of area method $(C O A)$ are two most common defuzzification methods, which $C O G$ method used the following equation to calculate the defuzzification

$$
\operatorname{COG}\left(x_{k}, y_{k}\right)=\frac{\sum_{i} U_{i} \sum_{j=1}^{r} \cdot \mu_{u}\left(x_{k}, y_{k}, U_{i}\right)}{\sum_{i} \sum_{j=1}^{r} \cdot \mu_{u}\left(x_{k}, y_{k}, U_{i}\right)}
$$

and $C O A$ method used the following equation to calculate the defuzzification

$$
\operatorname{COA}\left(x_{k}, y_{k}\right)=\frac{\sum_{i} U_{i} \cdot \mu_{u}\left(x_{k}, y_{k}, U_{i}\right)}{\sum_{i} \mu_{U} \cdot\left(x_{k}, y_{k}, U_{i}\right)}
$$

Where $\operatorname{COG}\left(x_{k}, y_{k}\right)$ and $\operatorname{COA}\left(x_{k}, y_{k}\right)$ illustrates the crisp value of defuzzification output, $U_{i} \in U$ is discrete element of an output of the fuzzy set, $\mu_{U} .\left(x_{k}, y_{k}, U_{i}\right)$ is the fuzzy set membership function, and $r$ is the number of fuzzy rules.

\section{Methodology}

The computed torque controller (CTC) has two main challenges:

- System's dynamic dependency

- Stability and robustness

To solve system's dynamic dependency, intelligent control theory is introduced. Fuzzy logic theory is one of the powerful intelligent method which used to estimate the challenge of dynamic dependency. Regarding to robot manipulator's dynamic formulation and CTC formulation:

$$
\begin{aligned}
& \tau=A(q) \ddot{q}+B(q)[\dot{q} \dot{q}]+C(q)[\dot{q}]^{2}+G(q) \\
& \tau=A(q)\left(\ddot{q}_{d}+K_{v} \dot{e}+K_{p} e\right)+B(q)[\dot{q} \dot{q}]+C(q)[\dot{q}]^{2}+G(q)
\end{aligned}
$$

Computed Torque Controller formulation is depending to robot manipulator's dynamic formulation. This controller has a fantastic response in certain condition but in uncertain situation it has many problem. To solve this important challenge fuzzy logic controller is applied in CTC. In this method; dynamic nonlinear equivalent part is replaced by performance/error-based PD like fuzzy logic controller. In this method; error based Mamdani's fuzzy inference system has considered with two inputs, one output and totally 49 rules instead of the dynamic equivalent part $\left(\boldsymbol{B}(\boldsymbol{q})[\dot{\boldsymbol{q}} \dot{\boldsymbol{q}}]+\boldsymbol{C}(\boldsymbol{q})[\dot{\boldsymbol{q}}]^{2}+\boldsymbol{G}(\boldsymbol{q})\right)$. Regarding to CTC formulation: 


$$
\hat{\tau}=\tau_{f u z z y}+A(q)\left(\ddot{q}_{d}+K_{v} \dot{e}+K_{p} e\right)
$$

Based on fuzzy logic methodology

$$
f(x)=U_{f u z z y}=\sum_{l=1}^{M} \theta^{T} \zeta(x)
$$

where $\boldsymbol{\theta}^{\boldsymbol{T}}$ is adjustable parameter (gain updating factor) and $\boldsymbol{\zeta}(\boldsymbol{x})$ is defined by;

$$
\zeta(x)=\frac{\sum_{i} \mu\left(x_{i}\right) x_{i}}{\sum_{i} \mu\left(x_{i}\right)}
$$

Where $\boldsymbol{\mu}\left(\boldsymbol{x}_{\boldsymbol{i}}\right)$ is membership function. $\boldsymbol{\tau}_{\boldsymbol{f} \boldsymbol{u z z} \boldsymbol{y}}$ is defined as follows;

$$
\tau_{f u z z y}=\sum_{l=1}^{M} \theta^{T} \zeta(x)=B(q)[\dot{q} \dot{q}]+C(q)[\dot{q}]^{2}+G(q)
$$

Based on above formulation, it has two inputs $(e, \dot{e})$ and one output $\left(\tau_{f u z z y}\right)$. The inputs are error (e) which measures the difference between desired and actual output position, and the change of error $(\dot{e})$ which measures the difference between desired and actual velocity and output is fuzzy equivalent torque. According to above algorithm to design the same PD controller the number of rule base for each link is $\boldsymbol{N} \times \boldsymbol{M}$. Where $\boldsymbol{N}$ is the number of linguistic variables for error and $\boldsymbol{M}$ is the number of linguistic variables for change of error.

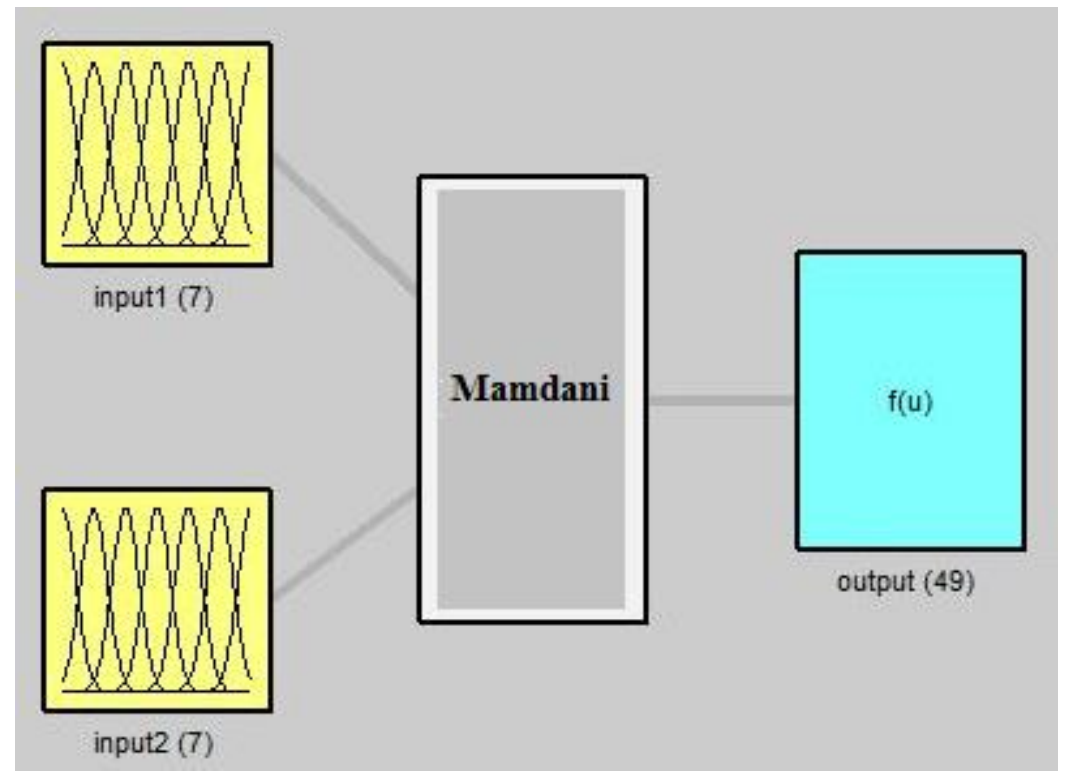

Figure 2. Fuzzy Inference System (FIS) Plot

The membership function plot for input 1 and input 2 show in Figure 3. 

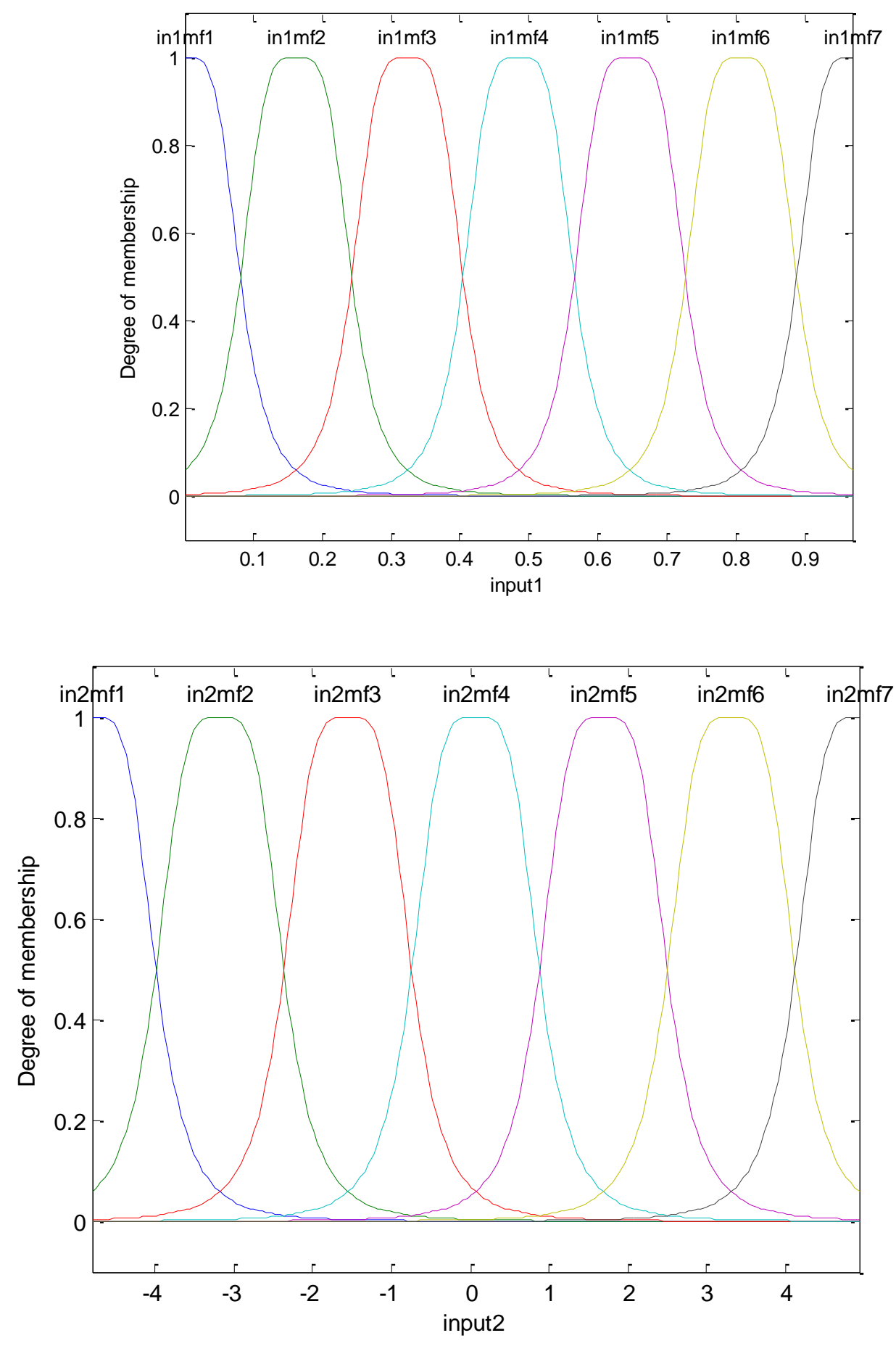

Figure 3. Membership Function Plot $(e$ and $\dot{e})$

Figure 4 shows the surface view in this research. 


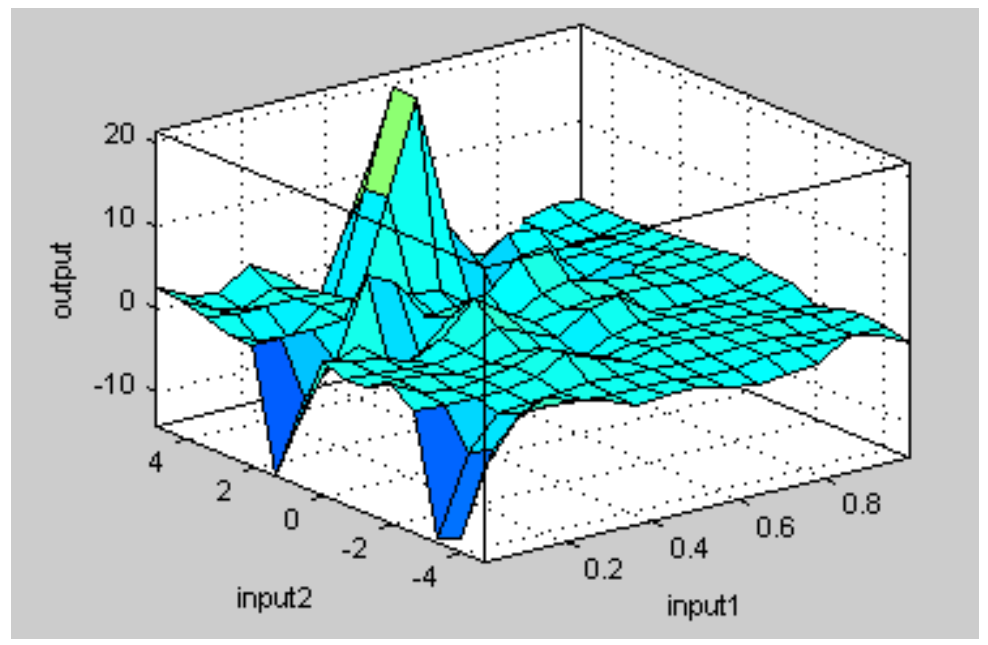

Figure 4. Surface View Plot

To tuning the membership functions and universe of discourse, in this research neurofuzzy is used and tune the PD like fuzzy logic controller. To improve the stability and robustness, in various dynamic parameters systems that need to be training on-line adaptive control methodology is used. In order to reduce the online computation burden, the PD like fuzzy logic controller is also used to coefficients online tuning. According to above, PD like fuzzy logic controller is used to increase the system stability in presence of system uncertainty.

$$
\begin{gathered}
U=\left(\ddot{q}_{d}+K_{v} \dot{e}+K_{p} e\right) \\
K_{p-\text { update }}=K_{p} \times U_{f u z z y} \\
K_{p-\text { update }}=K_{p} \times\left[\left(\sum_{l=1}^{M} \theta^{T} \zeta(x)\right)_{e, \dot{e}}\right]
\end{gathered}
$$

According to Lyapunov function, the formulation of lyapunov is;

$$
V=\frac{1}{2} \sum_{J=1}^{M} \frac{1}{\gamma_{j}} \phi^{T} . \phi_{j}
$$

$\boldsymbol{\phi}=\boldsymbol{\theta}^{*}-\boldsymbol{\theta}, \boldsymbol{\theta}^{*}$ is minimum error

$\theta$ is adjustable parameter

and the derivative of Lyapunov function $(\dot{\boldsymbol{V}})$ is

$\dot{V}=\frac{1}{2} \dot{A} \times N(q, \dot{q})+\sum_{J=1}^{M} \frac{1}{\gamma_{j}} \phi^{T} \cdot \dot{\phi}_{j}$

\section{Test and Result}

PD like fuzzy computed torque controller and adaptive PD like fuzzy computed torque controller are comparing in this part.

Comparison of the Tracking Data and Information: in certain condition, the data following for PD like fuzzy CTC and adaptive PD like fuzzy CTC are compared in this section. According to Figure 5, fuzzy CTC has about $15 \%$ overshoot in certain condition. In rise time point of view, PD like fuzzy CTC is better than adaptive PD like fuzzy CTC. 


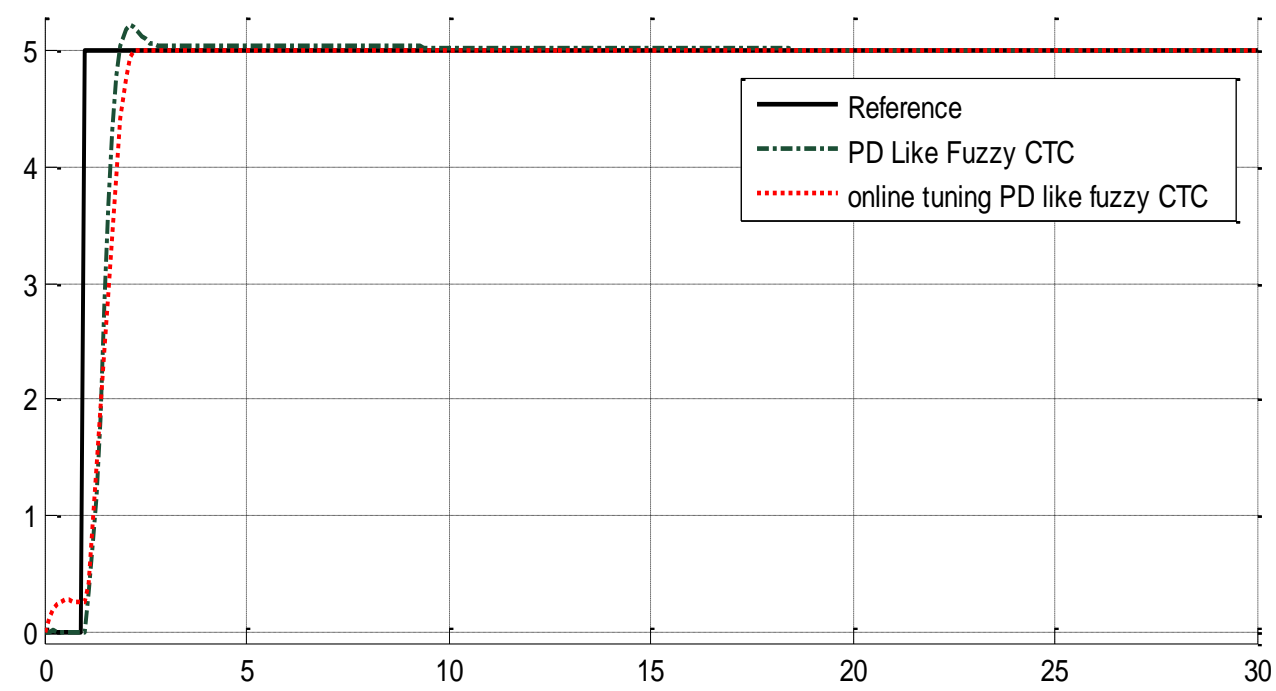

Figure 5. Tracking Data: PD Like Fuzzy CTC and Adaptive PD Like Fuzzy CTC

Robustness Checking: the power of disturbance rejection is very important to robust checking in any controllers. In this section trajectory accuracy is test under uncertainty condition. To test the disturbance rejection $30 \%$ band limited white noise applied to conventional above controllers. In Figure 9, trajectory accuracy performance is shown. According to Figure 6, PD like fuzzy controller has disturbance in presence of uncertainty, but adaptive PD like fuzzy CTC is stable in this situation. Regarding to Figure 6, rise time in fuzzy CTC is better than adaptive fuzzy CTC.

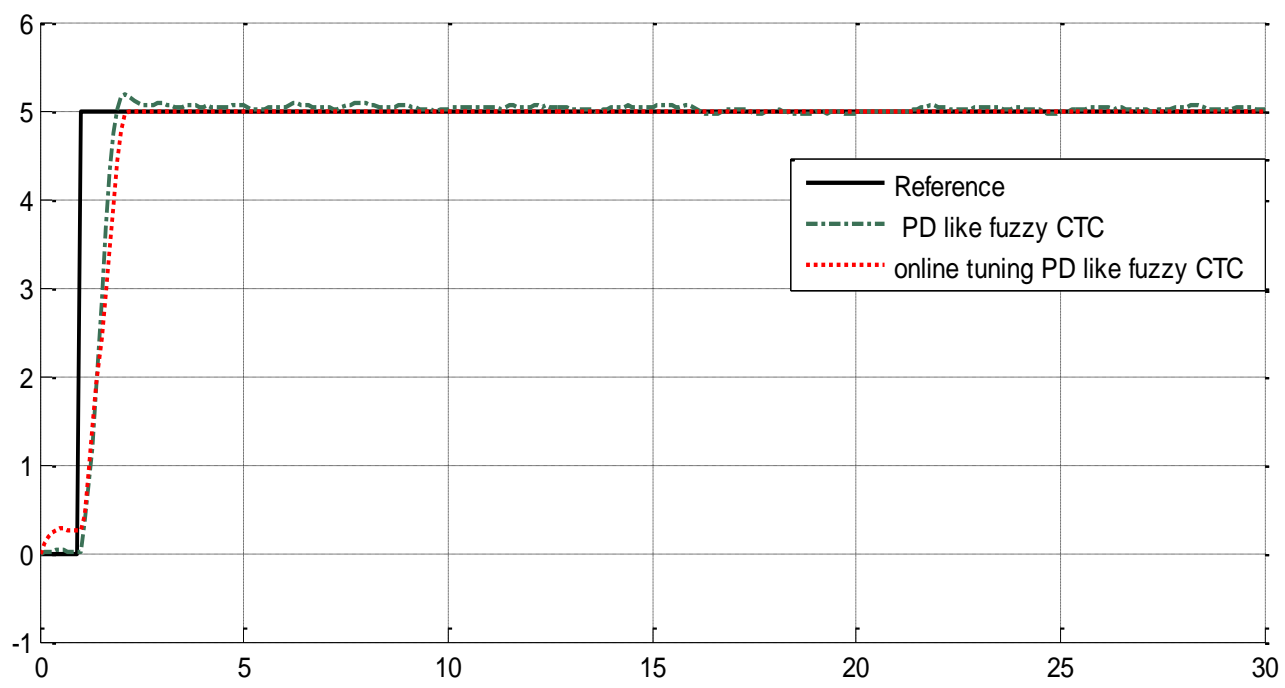

Figure 6. Tracking Data Disturbance Rejection: PD like Fuzzy CTC and Adaptive PD Like Fuzzy CTC

Tracking Error Comparison: in this part, tracking steady state error in fuzzy CTC and adaptive fuzzy CTC are compared. Figure 7 shows the steady state error in presence of uncertainties. According to this Figure, fuzzy CTC has moderate fluctuations in presence of uncertainty. In the following graph, the error rate of adaptive fuzzy CTC is near to the zero. 


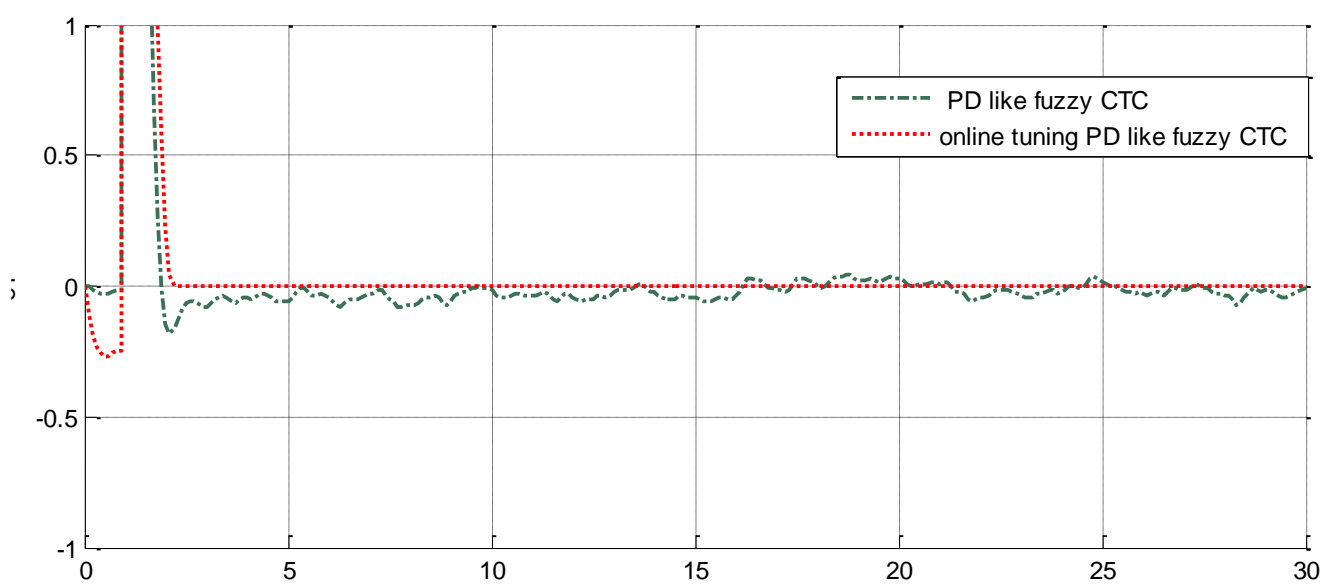

Figure 7. Steady State Error: PD like Fuzzy CTC and Adaptive PD like Fuzzy CTC

Figure 8 shows root means square (RMS) error in presence of uncertainty for PD like fuzzy CTC and adaptive PD like fuzzy CTC controller. Based on Figure 8, PID like fuzzy CTC has more position deviation.

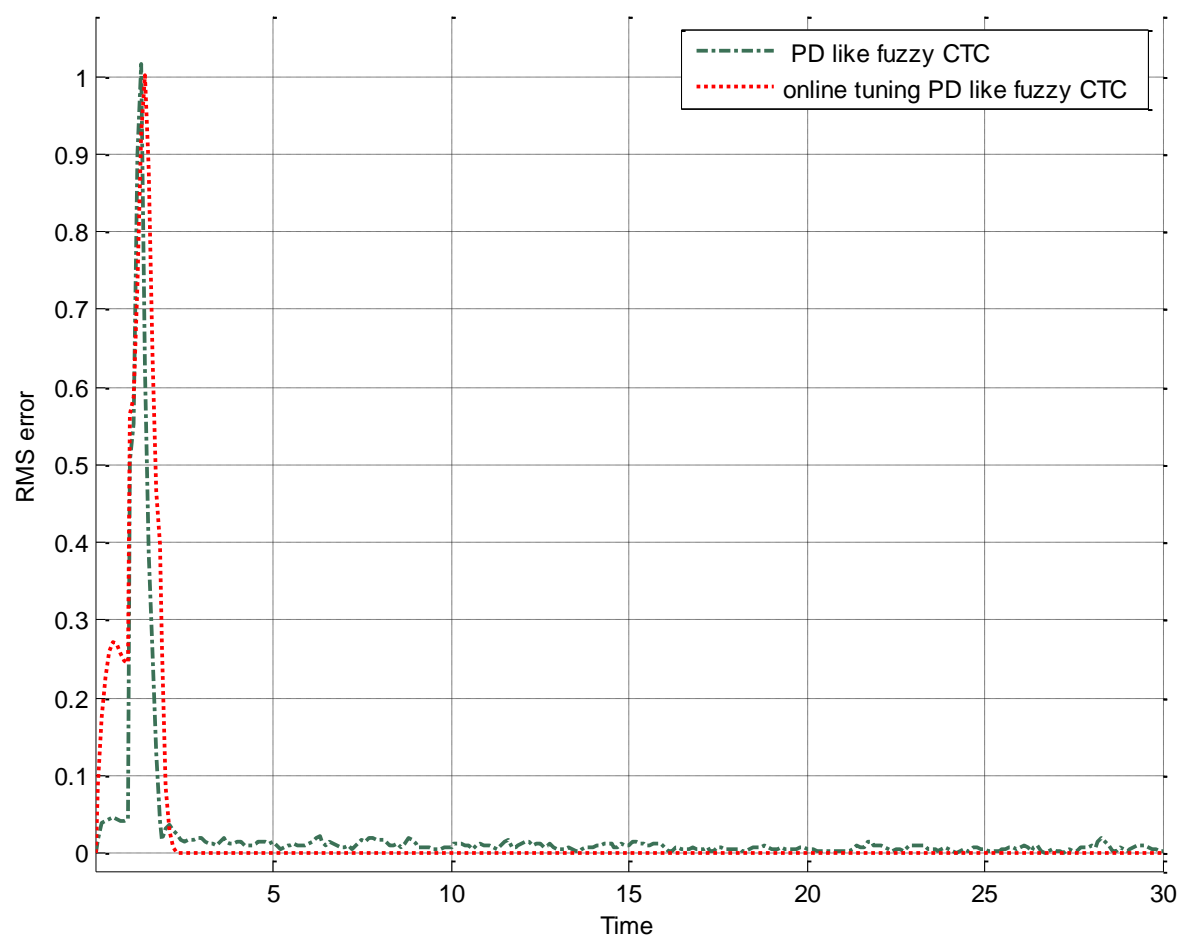

Figure 8. RMS Error: PD Like Fuzzy CTC and Adaptive PD Like Fuzzy CTC

\section{Conclusion}

Computed torque controller is a model base nonlinear controller. This controller has many advantages compare to linear controller. This controller has challenge in presence of uncertainty, to solve this challenge PD fuzzy logic controller with 49 bell-shape membership function is introduced. To improve the fuzzy results neuro-fuzzy is used. 
However, fuzzy CTC solve the challenge of dynamic dependency, it has the stability challenge. To solve the stability challenge, adaptive fuzzy CTC is introduced. The PD like fuzzy CTC is updated based on online tuning method. In order to reduce the online computation burden, the PD like fuzzy logic controller is also used to online tuning. As a result, in proposed method fuzzy logic controller is used to dynamic estimation and also online tuning. In proposed case of the $N$ degrees of freedom robot manipulator, if we defined $K$ membership functions for each input variable, the number of fuzzy rules for each joint of robot manipulator is $K^{2}$ which it is obviously decreased.

\section{Acknowledgment}

This work was supported by the Iranian Institute of Advance Science and Technology Program of Iran under grant no. 2015-Persian Gulf-2.

Project Title: Research on Intelligent FPGA-Based Algorithm for Four Degrees of Freedom Joints.

Iranian center of Advance Science and Technology (IRAN SSP) is one of the independent research centers specializing in research and training across of Control and Automation, Electrical and Electronic Engineering, and Mechatronics $\&$ Robotics in Iran. At IRAN SSP research center, we are united and energized by one mission to discover and develop innovative engineering methodology that solve the most important challenges in field of advance science and technology. The IRAN SSP Center is instead to fill a long standing void in applied engineering by linking the training a development function one side and policy research on the other. This center divided into two main units:

- $\quad$ Education unit

- $\quad$ Research and Development unit

Please follow IRANSSP research and training group: http://iranssp.org/english/

\section{References}

[1] F. Piltan, M. H. Yarmahmoudi, M. Shamsodini, E. Mazlomian and A. Hosainpour, "PUMA-560 Robot Manipulator Position Computed Torque Control Methods Using MATLAB/SIMULINK and Their Integration into Graduate Nonlinear Control and MATLAB Courses", International Journal of Robotics and Automation, vol. 3, no. 3, (2012), pp. 167-191.

[2] Vivas and V. Mosquera, "Predictive functional control of a PUMA robot", International Conference on Automatic Control, (2005), pp. 35-40.

[3] Z. Bingul. "Serial and Parallel Robot Manipulators - Kinematics, Dynamic"s, Control and Optimization. InTech, 14, 15, 17, 19, 20, 22, 49, 59, 60, 66, 67, (2012).

[4] Siciliano, B., \& Khatib, O. (Eds.), Springer handbook of robotics. Springer Science \& Business Media, (2008).

[5] Z. Song, , J. Yi, , D. Zhao and X. Li, A computed torque controller for uncertain robotic manipulator systems: Fuzzy approach. Fuzzy Sets and Systems, vol. 154, no. 2, (2005), pp. 208-226.

[6] J. T.. Jang, H. C. Gong and J. Lyou, Computed Torque Control of an aerospace craft using nonlinear inverse model and rotation matrix. In Control, Automation and Systems (ICCAS), 2015 15th International Conference on , (2015) (pp. 1743-1746) ,. IEEE.

[7] D. Choi and J. H. Oh, Active Suspension for a Rapid Mobile Robot Using Cartesian Computed Torque Control. Journal of Intelligent \& Robotic Systems, vol. 79, no. 2, (2015), pp. 221-235.

[8] S.Saidi, R. Abbassi and S. Chebbi,Fuzzy logic controller for three-level shunt active filter compensating harmonics and reactive power.International Journal of Adaptive Control and Signal Processing, (2015). 


\section{Authors}

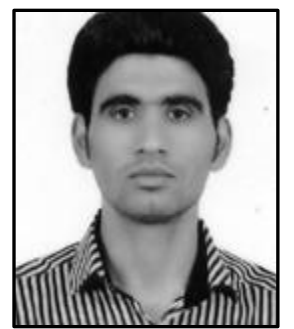

Hossein Rashidi Bod, he is currently is research student at Institute of Advanced Science and Technology, Research Center, IRAN SSP. He is research student of team (6 researchers) to design Micro-electronic Based nonlinear controller for Four Degrees of Freedom Surgical Robot Manipulator since August 2015. His current research interests are nonlinear control, artificial control system, Microelectronic Device, and HDL design.

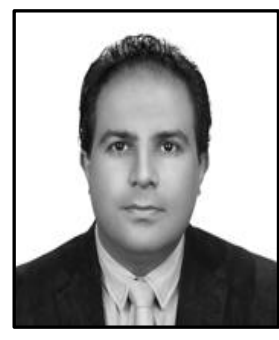

Farzin Piltan, he is an outstanding scientist in the field of Electronics and Control engineering with expertise in the areas of nonlinear systems, robotics, and microelectronic control. Mr. Piltan is an advanced degree holder in his field. Currently, Mr. Piltan is the Head of Mechatronics, Intelligent System, and Robotics Laboratory at the Iranian Institute of Advanced Science and Technology (IRAN SSP). Mr. Piltan led several high impact projects involving more than 150 researchers from countries around the world including Iran, Finland, Italy, Germany, South Korea, Australia, and the United States. Mr. Piltan has authored or coauthored more than 140 papers in academic journals, conference papers and book chapters. His papers have been cited at least 3900 times by independent and dependent researchers from around the world including Iran, Algeria, Pakistan, India, China, Malaysia, Egypt, Columbia, Canada, United Kingdom, Turkey, Taiwan, Japan, South Korea, Italy, France, Thailand, Brazil and more. Moreover, Mr. Piltan has peer-reviewed at least 23 manuscripts for respected international journals in his field. Mr. Piltan will also serve as a technical committee member of the upcoming EECSI 2015 Conference in Indonesia. Mr. Piltan has served as an editorial board member or journal reviewer of several international journals in his field as follows: International Journal of Control And Automation (IJCA), Australia, ISSN: 2005-4297, International Journal of Intelligent System and Applications (IJISA), Hong Kong, ISSN:2074-9058, IAES International Journal Of Robotics And Automation, Malaysia, ISSN:2089-4856, International Journal of Reconfigurable and Embedded Systems, Malaysia, ISSN:20894864.

Mr. Piltan has acquired a formidable repertoire of knowledge and skills and established himself as one of the leading young scientists in his field. Specifically, he has accrued expertise in the design and implementation of intelligent controls in nonlinear systems. Mr. Piltan has employed his remarkable expertise in these areas to make outstanding contributions as detailed follows:Nonlinear control for industrial robot manipulator (2010IRAN SSP), Intelligent Tuning The Rate Of Fuel Ratio In Internal Combustion Engine (2011-IRANSSP), Design High Precision and Fast Dynamic Controller For Multi-Degrees Of Freedom Actuator (2013-IRANSSP), Research on Full Digital Control for Nonlinear Systems (2011-IRANSSP), Micro-Electronic Based Intelligent Nonlinear Controller (2015-IRANSSP), Active Robot Controller for Dental Automation (2015-IRANSSP), Design a 
Micro-Electronic Based Nonlinear Controller for First Order Delay System (2015-IRANSSP).

The above original accomplishments clearly demonstrate that Mr. Piltan has performed original research and that he has gained a distinguished reputation as an outstanding scientist in the field of electronics and control engineering. Mr. Piltan has a tremendous and unique set of skills, knowledge and background for his current and future work. He possesses a rare combination of academic knowledge and practical skills that are highly valuable for his work. In 2011, he published 28 first author papers, which constitute about $30 \%$ of papers published by the Department of Electrical and Electronic Engineering at University Putra Malaysia. Additionally, his 28 papers represent about $6.25 \%$ and $4.13 \%$ of all control and system papers published in Malaysia and Iran, respectively, in 2011.

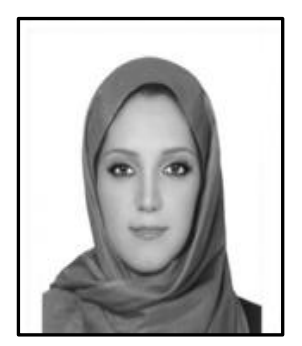

Somayeh Jowkar, she currently is research assistant at Institute of Advanced Science and Technology, Research Center, IRAN SSP. She is research assistant of team to Design Intelligent FPGA-Based Control Unit to Control of 4-DOF Medical Robot Manipulator since July, 2015 to now, research student (21 researchers) to design high precision and fast dynamic controller for multi-degrees of freedom actuator since 2014 to date, and published 2 journal papers since 2014 to date. Her current research interests are nonlinear control, artificial control system, Microelectronic Device and HDL design.

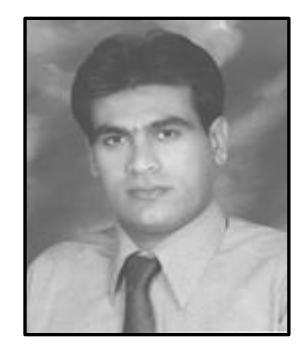

Amirzubir Sahamijoo, he is currently a senior research assistant at Institute of Advanced Science and Technology, Research Center, IRAN SSP. He is senior research assistant of team to Design Intelligent FPGA-Based Control Unit to Control of 4-DOF Medical Robot Manipulator since July, 2015 to now, research assistant of team (8 researchers) to design a Microelectronic Based nonlinear controller for first order delay system since March, 2015 to now, research student (21 researchers) to design high precision and fast dynamic controller for multi-degrees of freedom actuator since 2014 to date, research student (9 researchers) to design Prevent the Risk of Lung Cancer Progression Based on Fuel Ratio Optimization since 2014 to date, and published 4 journal papers since 2014 to date. His current research interests are nonlinear control, artificial control system, Microelectronic Device, Internal Combustion Engine, and HDL design.

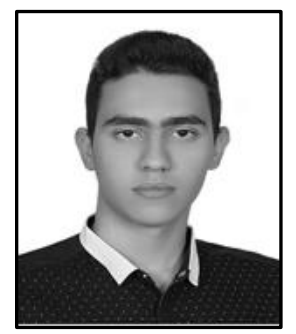

Ali Taghizadegan, he is currently studying as a student in the second grade of Shahid dastgheib's 1 high school and Research Student at Iranian Institute of Advanced Science and Technology, Research and Training Center, IRAN SSP. He is research student of team (6 researchers) to design Micro-electronic Based nonlinear controller for Four Degrees of Freedom Surgical Robot Manipulator since August 2015. His current research interests 
are nonlinear control, artificial control system, Microelectronic Device, and HDL design.

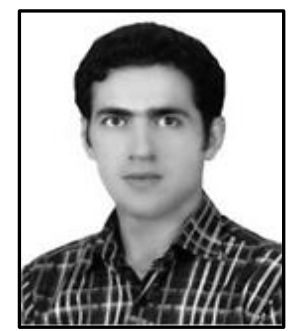

Rouhollah Bahrami, he is currently a research student at Institute of Advanced Science and Technology, Research Center, IRAN SSP. He is research student of team (6 researchers) to design Micro-electronic Based nonlinear controller for Four Degrees of Freedom Surgical Robot Manipulator since August 2015. His current research interests are nonlinear control, artificial control system, Microelectronic Device, and HDL design.

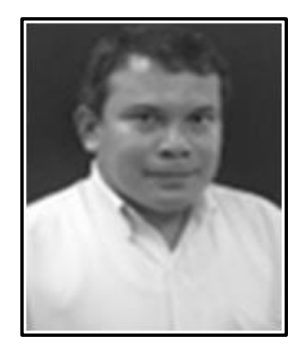

Nasri Sulaiman, he is an adviser and supervisor of several high impact projects involving more than 150 researchers from countries around the world including Iran, Malaysia, Finland, Italy, Germany, South Korea, Australia, and the United States. Dr. Nasri Sulaiman has authored or co-authored more than 80 papers in academic journals, conference papers and book chapters. His papers have been cited at least 3000 times by independent and dependent researchers from around the world including Iran, Algeria, Pakistan, India, China, Malaysia, Egypt, Columbia, Canada, United Kingdom, Turkey, Taiwan, Japan, South Korea, Italy, France, Thailand, Brazil and more. Dr. Nasri Sulaiman has employed his remarkable expertise in these areas to make outstanding contributions as detailed below:

- Design of a reconfigurable Fast Fourier Transform (FFT) Processor using multi-objective Genetic Algorithms (2008-UPM)

- Power consumption investigation in reconfigurable Fast Fourier Transform (FFT) processor (2010-UPM)

- Crest factor reduction And digital predistortion Implementation in Orthogonal frequency Division multiplexing (ofdm) systems (2011-UPM)

- High Performance Hardware Implementation of a MultiObjective Genetic Algorithm, (RUGS), Grant amount RM42,000.00, September (2012-UPM)

- Nonlinear control for industrial robot manipulator (2010-IRAN SSP)

- Intelligent Tuning The Rate Of Fuel Ratio In Internal Combustion Engine (2011-IRANSSP)

- Design High Precision and Fast Dynamic Controller For MultiDegrees Of Freedom Actuator (2013-IRANSSP)

- Research on Full Digital Control for Nonlinear Systems (2011IRANSSP)

- Micro-Electronic Based Intelligent Nonlinear Controller (2015IRANSSP)

- Active Robot Controller for Dental Automation (2015-IRANSSP)

- Design a Micro-Electronic Based Nonlinear Controller for First Order Delay System (2015-IRANSSP) 
International Journal of Control and Automation

Vol. 9, No. 9 (2016) 